\title{
Local Color Mapping Combined with Color Transfer for Underwater Image Enhancement
}

\author{
Rafał Protasiuk \\ Adel Bibi \\ Warsaw University of Technology, \\ King Abdullah University of Science and Technology, \\ r.protasiuk@ire.pw.edu.pl \\ adel.bibiekaust.edu.sa \\ Bernard Ghanem \\ King Abdullah University of Science and Technology \\ bernard.ghanemekaust.edu.sa
}

\begin{abstract}
Color correction and color transfer methods have gained a lot of attention in the past few years to circumvent color degradation that may occur due to various sources. In this paper, we propose a novel simple yet powerful strategy to profoundly enhance color distorted underwater images. The proposed approach combines both local and global information through a simple yet powerful affine transform model. Local and global information are carried through local color mapping and color covariance mapping between an input and some reference source, respectively. Several experiments on degraded underwater images demonstrate that the proposed method performs favourably to all other methods including ones that are tailored to correcting underwater images by explicit noise modelling.
\end{abstract}

\section{Introduction}

The colors that are present in images captured by a camera are mostly determined by scene illumination introduced by a light source. In extreme conditions - like underwater imaging - colors could be profoundly affected by distorted light propagation due to the specific wavelengths absorption, which results in scenes with visible blue/green dominated color casting $[1,2,17]$. Such color casts are perceptually undesirable and have adverse effects on subsequent processing such as object recognition and tracking since it affects overall color pixels values of an image. For a robust color-based system e.g. tracking, image segmentation and $3 \mathrm{D}$ reconstruction, these effects should be corrected.

Local color mapping methods $[4,12,14,19]$ in general rely on obtaining a map between a few selected colors and their ground truth. However, the results of such approaches are not satisfactory as they lack access to global information generating images that are not natural. This is due to the fact that such methods do not respect overall color distribution. On the other hand, color transfer methods $[7,8,21,22,23]$ utilize global information from the image. Simple approaches tend to map mean and the standard deviation from a reference image. This approach is known to be effective in many contexts. However, color transfer based methods are not well suited to the spatially variable color casts encountered in highly color distorted images as in underwater scenes. This is since such methods solely rely on global image statistics ignoring to correct colors locally.

In this paper, we introduce a novel color correction method which combines the best of local color mapping and color transfer approaches through a single affine model (Figure 1) that can be easily propagated along sequences of images from the same scene. We perform a comprehensive qualitative and quantitative evaluation against several types of techniques. In addition to its simplicity, our method outperforms the previous state of the art on extreme underwater cases of a large color distortion.

The paper is structured as follows. Section 2 describes related works; Section 3 overviews our proposed objective function that combines through an affine model both the local color and global covariance mappings. In section 4 , we demonstrate with several experiments on several underwater images that our proposed modelling outperforms several state-of-the-art methods. We lastly conclude the paper with a summary and a discussion in Section 5.

\section{Related work}

In this section, we highlight different previous methods for underwater color correction along with the work on color transfer which is related to our approach.

Underwater Color Correction. Most of the recent approaches designed to restore underwater images rely on un- 


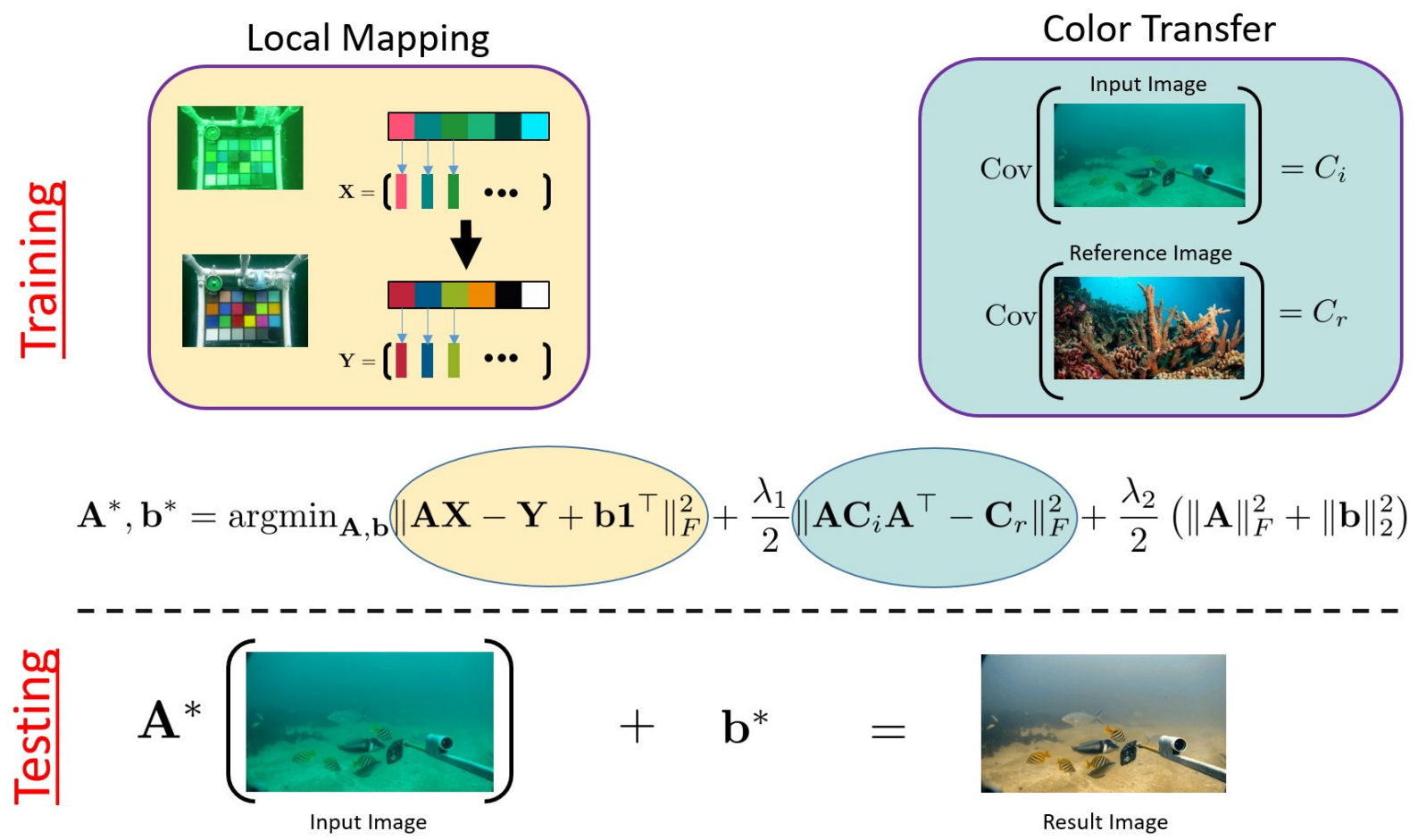

Figure 1. Shows the proposed pipeline divided between local mapping and color transfer used to estimate the parameters. In the testing, the affine model is simply applied to the input image.

derwater camera model that has a similar form as the optical model of Koschmieder [15] which was used to characterize the propagation of light in the atmosphere. A representative example is Chiang et al. [3] method, that estimates the rough depth map of the underwater scene based on the Dark Channel Prior (DCP) - initially introduced by He et al. [13] for outdoor dehazing. DCP assumes that the radiance of an object in a natural scene is small in at least one of the color component and consequently defines regions of small transmission as the ones with large minimal value of colors. There have been also multiple proposals for nonoptical model-based methods. Garcia et al. [11] proposed local histogram equalization to enhance underwater scenes with non-uniform lighting. Inspired by HDR imaging, Ancuti et al. [1] proposed a method that merges two color corrected images using a multi-scale fusion technique. Galdran et al. [10] proposed a red channel-based underwater image restoration method. Drews-Jr et al. [5] assumed that the predominant source of visual information under the water lies in the blue and green color channels and introduced the new prior - Underwater Dark Channel Prior (UDCP). All the briefly discussed methods focus on eliminating distortions caused by haze effect and neglect color casting effects; thereafter, the resultant images improve in sense of contrast and visibility; however, the corrected images still lack the realistic range of colors that are in natural images. On the other hand, recent approaches that use generative models, specifically Generative Adversarial Networks (GANs) towards generating underwater images $[6,16]$ results in very realistic images, but the process of training such models creates a need for extensive computational resources.

Color Transfer. The earliest work on color transfer is the work of Reinhard et al. [22] who has introduced a simple and efficient framework, which matches the means and the standard deviations of the global color distributions of the two images in the LAB color space. Pitie et al. [20] presented a color transfer method based on the iterative use of a 1D histogram matching preceded by usage of 3D rotation matrix to rotate the source and the target images to a certain axis in color space. Xiao and Ma [24] proposed to handle color transfer in the RGB space by using linear transforms (translation, scale, and rotation) to align the two gamuts of the source and target images. In [25], Xiao and Ma extended their work to map the histograms of two images together and included a step to preserve the gradient of the source image. Nguyen et al. [18] introduced method that put constraint on the mapped image that must be within the color gamut of the target image, by performing a gradient preserving color histogram matching. Although color transfer methods are not widely used for image color correction, their ability to enhance colors can improve highly distorted images with proper references. 


\section{Proposed approach}

Most previous methods rely on either the local color mapping or global color transfer for color enhancement in general but not both jointly. In this segment, we explain our proposed model that can combine both approaches in a surprisingly straightforward yet powerful approach. We adopt the simple affine model $\mathbf{y}=\mathbf{A} \mathbf{x}+\mathbf{b}$ that maps a given input pixel $\mathbf{x} \in \mathbb{R}^{3 \times 1}$ to the output $\mathbf{y} \in \mathbb{R}^{3 \times 1}$ through a simple affine transform consisting of $\mathbf{A} \in \mathbb{R}^{3 \times 3}$ and $\mathbf{b} \in \mathbb{R}^{3 \times 1}$. As for the global color transfer, and following previous work, this is often achieved by transferring global information from some predetermined reference images that have the desired global color distribution. To achieve this, we propose a new regularizer that matches covariances between the output transformed image through the affine transform and the covariance of the reference source image. Thus, our proposed objective that we seek to solve is given as follows:

$$
\begin{array}{rl}
\min _{\mathbf{A}, \mathbf{b}} & f(\mathbf{A}, \mathbf{b}) \stackrel{\text { def }}{=} \underbrace{\left\|\mathbf{A} \mathbf{X}-\mathbf{Y}+\mathbf{b} \mathbf{1}_{3}^{\top}\right\|_{F}^{2}}_{\text {Local Color Mapping }}+ \\
\frac{\lambda_{1}}{2} \overbrace{\left\|\mathbf{A} \mathbf{C}_{i} \mathbf{A}^{\top}-\mathbf{C}_{r}\right\|_{F}^{2}}^{\text {Global Color Transfer }}+\frac{\lambda_{2}}{2}\left(\|\mathbf{A}\|_{F}^{2}+\|\mathbf{b}\|_{2}^{2}\right)
\end{array}
$$

It is clear from the proposed optimization that we seek to balance out the one-to-one color mapping against a global covariance matching with a source reference. We also add another prior on the energy of the affine model to keep it bounded. Such term will be vital for some specific applications. In what follows, we will discuss each of the three terms thoroughly.

Local Color Mapping. In many scenarios, the true colors of some corrupted pixels are known apriori. For instance, underwater images are naturally distorted. The true colors of some pixels may be know when a dedicated color board is available in scenes (for above water and underwater scenarios). The known colors are in general very sparse depending on the application. The local color mapping term in this setting $\left\|\mathbf{A X}-\mathbf{Y}+\mathbf{b}_{3}^{\top}\right\|_{F}^{2}$ matches the $N$ selected pixels, where $N$ is the number of columns in $\mathbf{X}$ and $\mathbf{Y}$.

Global Color Transfer. In many previous works, transferring high order statistics [23, 24] like the variances from some reference images helps in transferring some global knowledge from a given reference source. However, these sort of approaches still can not capture the inter-correlation among pixels limiting their performance. However, under the simple proposed affine model $\mathbf{y}=\mathbf{A x}+\mathbf{b}$ such transformation is now possible. Since the output image under the affine map has the following color covariance $\mathbf{A C}_{i} \mathbf{A}^{\top}$ where $\mathbf{C}_{i}$ is the color covariance of the input image, we propose the regularizer $\left\|\mathbf{A C}_{i} \mathbf{A}^{\top}-\mathbf{C}_{r}\right\|$ that matches the two covariances together. The reference color covariance $\mathbf{C}_{r}$ is often know apriori or can be statistically collected from a set of natural images.

Prior term. Only introducing the previously discussed regularizers $\left\|\mathbf{A X}-\mathbf{Y}+\mathbf{b} \mathbf{1}^{\top}\right\|_{F}^{2}+\frac{\lambda_{1}}{2}\left\|\mathbf{A} \mathbf{C}_{i} \mathbf{A}^{\top}-\mathbf{C}_{r}\right\|_{F}^{2}$ may still lead to instability in performance. This is because in many degraded settings, the red channel in underwater images may be washed out completely and almost non existent in the input image $\mathbf{X}$. This happens particularly in images taken deep underwater, image acquisition under such circumstances suffers extreme attenuation in the red channel resulting into pixel values to be very close to zero. Only using the regularizers above may result into having very high energy for the affine model parameter in trying to match the washed red pixel values to follow the the local mapping or the global red pixel covariance. This will result into artifacts saturating the output image into one color. Figure 2 demonstrates an example of an image taken underwater and the effect of introducing such a prior term. Thus, to keep the energy of the affine transform bounded, we add another regularizer $\|\mathbf{A}\|_{F}^{2}+\|\mathbf{b}\|_{2}^{2}$ that resolves this nuisance.

Solver. Problem (1) is not convex due to the $4^{\text {th }}$ order polynomial $\mathbf{A} \mathbf{C}_{i} \mathbf{A}^{\top}$; however, the objective is smooth. We simply solve the problem using gradient descent. Using a matrix variation approach, we derive the gradient of the non convex part. The detailed derivation of the gradients of all the terms can be found in the supplementary material.

Proposition 1 The gradients of (1) with respect to the parameters $\mathbf{A}$ and $\mathbf{b}$ are given as follows:

$$
\begin{gathered}
\nabla_{\mathbf{A}} f(\mathbf{A}, \mathbf{b})=\lambda_{1}\left(\mathbf{A X}-\mathbf{Y}+\mathbf{b} \mathbf{1}^{T}\right) \mathbf{X}^{T}+2 \lambda_{1} \mathbf{A} \mathbf{C}_{i} \mathbf{A}^{T} \mathbf{A} \mathbf{C}_{i} \\
-2 \lambda_{1} \mathbf{C}_{r} \mathbf{A} \mathbf{C}_{i}+\lambda_{2} \mathbf{A} \\
\nabla_{\mathbf{b}} f(\mathbf{A}, \mathbf{b})=\lambda_{1}\left(\mathbf{A X}-\mathbf{Y}+\mathbf{b} \mathbf{1}^{T}\right) \mathbf{1}+\lambda_{2} \mathbf{b}
\end{gathered}
$$

\section{Experiments}

In this section, we conduct two different experiments to demonstrate the effectiveness and robustness of the proposed method. We start by demonstrating that our objective function (1) strongly correlates with how people perceive natural images Exp.(1). Then we compare our proposed method against state-of-the-art methods on a set of naturally distorted underwater images Exp.(2). Moreover, to guarantee the fairness of the experiments we compare our method against all other methods with and without the local color mapping term.

Parameters and Implementation Details. We conduct our experiments on a dataset of 50 manually collected images distorted naturally underwater with the companion of pleasant looking underwater reference images. As for the color correspondences, a set of precomputed correspondences was made and used throughout the experiments. For the fairness of the experiments, all methods used the same 


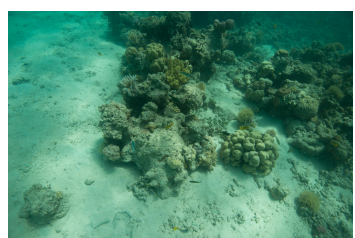

Input

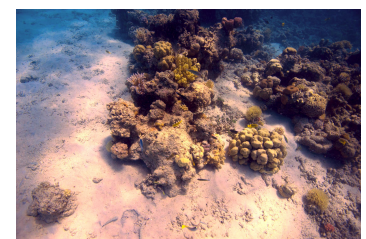

$\lambda_{1}, \lambda_{2}=0$

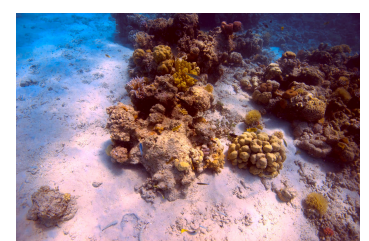

$\lambda_{1}=1 \quad \lambda_{2}=0$

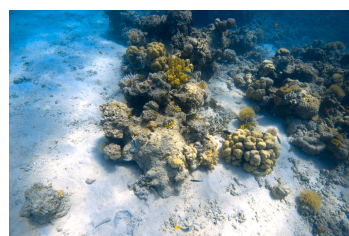

$\lambda_{1}=1 \quad \lambda_{2}=1$

Figure 2. Shows qualitative measure with varying values of $\lambda_{1}$ and $\lambda_{2}$. It is evident that eliminating the energy term by setting $\lambda_{2}=0$, the resultant image is dominated with the red channel. See text for details.

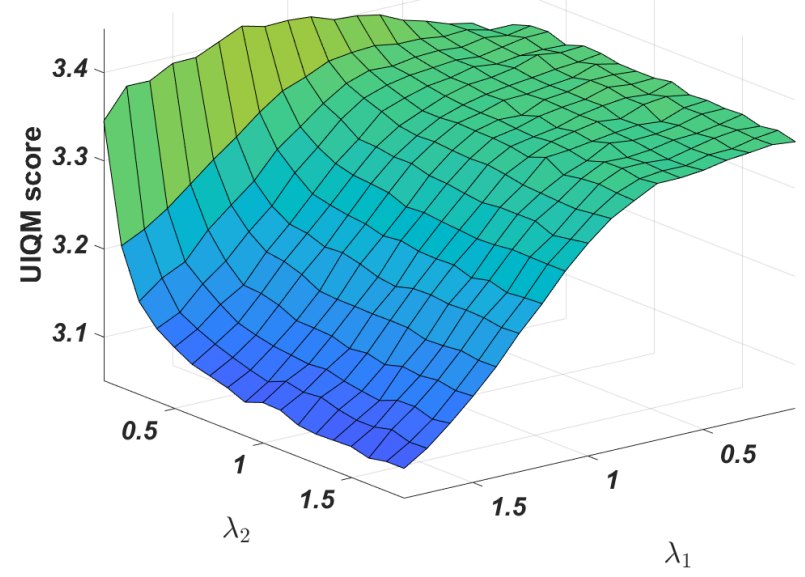

Figure 3. Shows quantitative results described by the UIQM measure computed on an underwater image with varying values of $\lambda_{1}$ and $\lambda_{2}$.

references and color correspondences throughout the comparisons. As for the first and second experiments where no ground truth is available for the underwater images, we conduct the experiments on Amazon Mechanical Turk (AMT) platform for subjective results. Each experiment was held on 10 unique turkers for evaluation per decision sample. Moreover, and for fair comparison, we introduce the color mapping term $\left\|\mathbf{A X}-\mathbf{Y}+\mathbf{b} \mathbf{1}^{\top}\right\|_{F}^{2}$ to every method that does not take in consideration the local color map by solving the following optimization problem.

$$
\min _{\mathbf{A}, \mathbf{b}}\left\|\mathbf{A X}-\mathbf{Y}+\mathbf{b} \mathbf{1}^{T}\right\|_{F}^{2}+\left\|\mathbf{A I}-\mathbf{J}_{i}+\mathbf{b} \mathbf{1}^{T}\right\|_{F}^{2}
$$

The input image intensity for a method $i$ is $\mathbf{I} \in \mathbb{R}^{3 \times n}$, where $\mathrm{n}$ is the number of pixels. As for $\mathbf{J} \in \mathbb{R}^{3 \times n}$, it denotes the pixel values as resulted from method $i$. Such a problem will simply find an affine model $\mathbf{A}, \mathbf{b}$ that will incorporate the color correspondence.

Justifying Design Parameters. To justify the model and the influence of each term in the objective function, we vary the weights of $\lambda_{1}$ and $\lambda_{2}$ to see their impact both qualitatively and quantitatively. We conduct two different experiments, one where we vary the values of $\lambda \mathrm{s}$ between the two end of extremes $(0,1)$ to showcase the impact qualitatively on the resultant image. The other experiment where we vary the values of both $\lambda$ s continuously and report and the objective measure (UIQM) of the underwater color corrected image. The UIQM metric [26] specialises on evaluating underwater color reconstruction methods (the larger the metrics, the better the quality). The UIQM comprises three underwater image attribute measures: the underwater image colourfulness measure (UICM), the underwater image sharpness measure (UISM), and the underwater image contrast measure (UIConM), all of them inspired by the properties of human visual system and developed basing on subjective experiments.

As for the first experiment, figure 2 demonstrates the role of the energy term in the objective in the underwater setting. This is as discussed earlier due to the fact that most underwater images have a washed out red channel. This will result in large values in the parameters $\mathbf{A}$ and $\mathbf{b}$ saturating the resultant image with red colors as seen in figure 2 . As for the figure 3 and comparing to case where both have a value of 0 , keeping $\lambda_{2}$ close to 0 and increasing $\lambda_{1}$ increases UIQM score, which results in higher quality image. This justifies the importance of global color transfer on the overall performance. Also, setting both parameters to high values will result in degraded performance. This is because the energy term $\|\mathbf{A}\|_{F}^{2}+\|\mathbf{b}\|_{2}^{2}$ is dominated resulting into transformations $\mathbf{A}$ and $\mathbf{b}$ that are very close to 0 . Based on the initial checking we achieved best results when setting $\lambda_{1}=0.75$ and $\lambda_{2}=0.05$ and these values were used as final in all conducted experiments.

Experiment (1): The Quality of the Proposed Objective Function. To validate that our proposed objective reflects the actual human perception of natural images, we conduct the following experiment on AMT. The experiment setup goes by asking each user to rank 4 images on a scale from 1 (least realistic) to 4 (most realistic). The images presented are the solutions at different iterations of the gradient descent. For instance, figure 4 showcases different solutions to the optimization problem at different iterations. If the objective function was in fact correlated to how people perceive natural images, then users will vote on images that have the least objective function as the most realistic, and vice versa. Table (1) presents the average scores for 


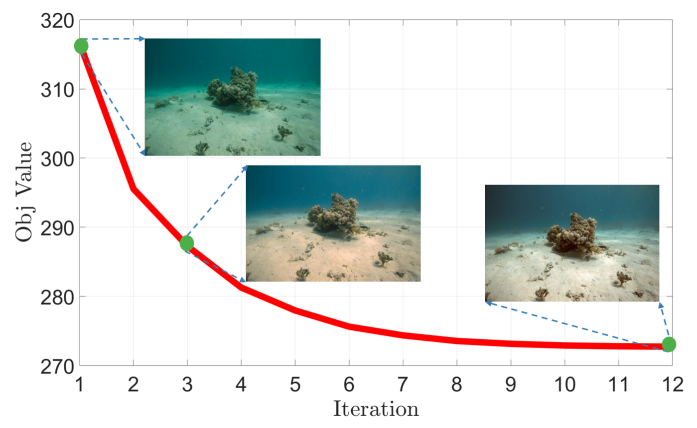

Figure 4. Demonstrate the objective function value decreases as the quality of the mid-iteration resultant images improves.

each consequent iteration step averaged over 50 underwater image examples voted among over 10 users per image. It is evident from table (1) that in fact our objective function captures some human perception of how people perceive natural images. This is due to that images that are generated with lower objective value (higher iteration step count) have higher voting score.

\begin{tabular}{|c|cccc|}
\hline Iteration step & $\# 1$ & $\# 2$ & $\# 3$ & $\# 4$ \\
\hline $\mathbb{E}$ & 1.70 & 2.44 & 2.79 & 3.07 \\
$\sigma$ & 1.05 & 0.97 & 0.94 & 0.99 \\
\hline
\end{tabular}

Table 1. Lists the average and standard deviation vote of 10 turkers over 50 underwater images. The higher the iteration step the lower the objective, where the higher the average score the closer it is to being realistic.

Experiment (2): Comparison Against the State of the Art on Underwater Images. In this experiment we compare our proposed method against four generic color transfer methods [18, 20, 22, 25], four methods that are tailored for correcting underwater images $[1,5,9,6]$ by using light attenuation models, and against Auto-Color option provided by Photoshop. The experiment is conducted on 50 underwater images using both subjective and objective metrics. As for the subjective metric, we conduct AMT experiments where we present the turkers with two images at a time where one is the result of our proposed method and the other is from one of the other methods. Since none of these methods compared against make use of any local color mapping, we compare our proposed method with two versions of each method. A comparison against the vanilla version of each method, and another against the solution to problem (2) for that method. In table (2), we list the comparison results where we report the average number of times users chose the other method as a more realistic image. Therefore, the lower the average the better the proposed approach over the competitor. It is also interesting to note that almost all methods in fact improved in performance after solving equation (2) motivating the importance of combining both local and

\begin{tabular}{ccc|cc}
\hline \multirow{2}{*}{ Method } & \multicolumn{2}{c|}{ After Solving (2) } & \multicolumn{2}{c}{ Before Solving (2) } \\
& $\mathbb{E}[$ ratio] & $\sigma$ & $\mathbb{E}[$ ratio] & $\sigma$ \\
\hline \hline Reinhard et al. [22] & 0.41 & 0.458 & 0.24 & 0.326 \\
Pitie et al. [20] & 0.40 & 0.436 & 0.36 & 0.368 \\
Xiao et al. [25] & 0.33 & 0.389 & 0.17 & 0.285 \\
Nguyen et al. [18] & 0.42 & 0.474 & 0.29 & 0.359 \\
\hline Ancuti et al. [1] & 0.45 & 0.350 & 0.35 & 0.320 \\
Fu et al. [9] & 0.40 & 0.312 & 0.31 & 0.342 \\
Drews et al. [5] & 0.43 & 0.512 & 0.37 & 0.512 \\
Fabbri et al. [6] & 0.52 & 0.331 & 0.43 & 0.231 \\
\hline Photoshop & 0.49 & 0.385 & 0.38 & 0.492 \\
\hline
\end{tabular}

Table 2. Lists comparisons between our method against the stateof-art. The table reports the average ratio between a given method and ours. The lower the value the better our method's performance. The first four methods are generic color transfer where the before last four methods are underwater tailored methods (with exclusion of Photoshop).

\begin{tabular}{ccc}
\hline \multirow{2}{*}{ Method } & \multicolumn{2}{c}{$\mathrm{UIQM}(\nearrow)$} \\
& $\mathbb{E}$ & $\sigma$ \\
\hline \hline Reinhard et al. [22] & 2.686 & 1.543 \\
Pitie et al. [20] & 2.590 & 1.491 \\
Xiao et al. $[25]$ & 2.753 & 1.382 \\
Nguyen et al. [18] & 2.528 & 1.426 \\
\hline Ancuti et al. [1] & 2.156 & 1.928 \\
Fu et al. $[9]$ & 3.892 & 1.026 \\
Drews et al. [5] & 2.492 & 1.146 \\
Fabbri et al. $[6]$ & 4.012 & 1.085 \\
\hline Photoshop & 2.469 & 1.058 \\
\hline Ours & 2.858 & 1.536 \\
\hline
\end{tabular}

Table 3. Lists comparisons between our method against the stateof-art with respect to UIQM metric scores. The higher the value the better performance.

global information. We also list some qualitative results for comparison in figure 6 . As for the objective comparison, another experiment is conducted with the same comparisons; however, the UIQM metric $(\nearrow)$ [26] is used in the comparison. Table 3 lists all the comparisons. Our proposed approach outperforms all generic color transfer methods even some underwater specific methods [1]. Despite the simplicity of the proposed approach it is still competitive with some methods that use dedicated full modeling of the underwater light models [9] or extensive generative neural networks models [6].

In general such simple powerful color correction scheme can help various computer vision tasks e.g. 3D point cloud generation. Figure 5, showcases different snapshots of the point cloud before and after the color correction. The 3D video and some more results are presented in the supplementary material. 

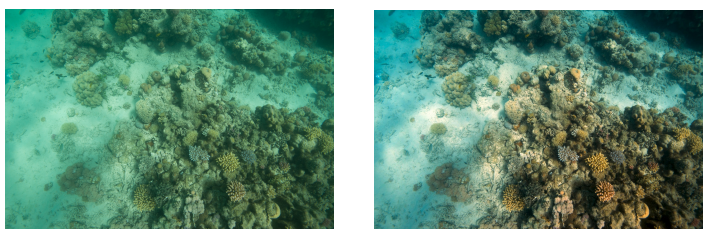

Sample input image
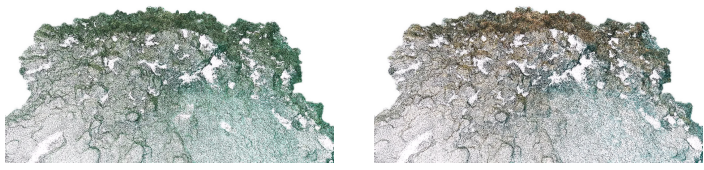

Dense 3D points map
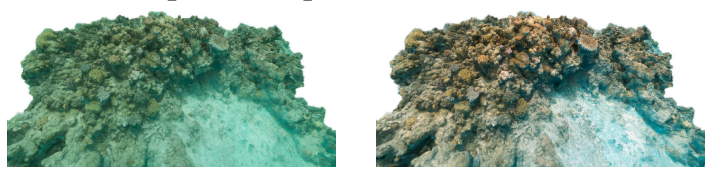

Textured 3D model

Figure 5. Presents reef 3D model reconstruction example using Structure from Motion techniques. Fist row presents results with regular naturally distorted underwater images, second row showcases same case for corrected ones.

\section{Conclusions}

In this paper, we introduced a simple yet powerful robust color correction method underwater images. The method combines the best of both of color mapping and color transfer based methods. Several experiments were conducted both qualitatively and quantitatively comparing our method to several other state-of-the-art methods. Our proposed method achieved very competitive and sometimes state-ofthe-art performance overcoming some other underwater tailored methods.

Acknowledgements. This work was supported by the King Abdullah University of Science and Technology Office of Sponsored Research.

\section{References}

[1] C. Ancuti, C. O. Ancuti, T. Haber, and P. Bekaert. Enhancing underwater images and videos by fusion. In IEEE Conference on Computer Vision and Pattern Recognition, pages 81-88, 2012.

[2] C. O. Ancuti, C. Ancuti, C. De Vleeschouwer, and R. Garcia. A semi-global color correction for underwater image restoration. In ACM SIGGRAPH, pages 66:1-66:2, 2017.

[3] J. Y. Chiang and Y. C. Chen. Underwater image enhancement by wavelength compensation and dehazing. IEEE Transactions on Image Processing, 21(4):1756-1769, 2012.

[4] C. Doutre and P. Nasiopoulos. Color correction preprocessing for multiview video coding. IEEE Transactions on Circuits and Systems for Video Technology, 19(9):1400-1406, 2009.

[5] P. L. J. Drews, E. R. Nascimento, S. S. C. Botelho, and M. F. M. Campos. Underwater depth estimation and image restoration based on single images. IEEE Computer Graphics and Applications, 36(2):24-35, 2016.

[6] C. Fabbri, M. J. Islam, and J. Sattar. Enhancing Underwater Imagery using Generative Adversarial Networks. In IEEE International Conference on Robotics and Automation, 2018.

[7] H. S. Faridul, T. Pouli, C. Chamaret, J. Stauder, E. Reinhard, D. Kuzovkin, and A. Tremeau. Colour Mapping: A Review of Recent Methods, Extensions and Applications. Computer Graphics Forum, 35(1):59-88, 2016.

[8] H. S. Faridul, J. Stauder, J. Kervec, and A. Trémeau. Approximate cross channel color mapping from sparse color correspondences. In IEEE International Conference on Computer Vision Workshops, pages 860-867, 2013.

[9] X. Fu, P. Zhuang, Y. Huang, Y. Liao, X. P. Zhang, and $X$. Ding. A retinex-based enhancing approach for single underwater image. In IEEE International Conference on Image Processing, pages 4572-4576, 2014.

[10] A. Galdran, D. Pardo, A. Picón, and A. Alvarez-Gila. Automatic Red-Channel underwater image restoration. Journal of Visual Communication and Image Representation, 26:132 $-145,2015$.

[11] R. Garcia, T. Nicosevici, and X. Cufi. On the way to solve lighting problems in underwater imaging. In OCEANS '02 MTS/IEEE, volume 2, pages 1018-1024 vol.2, 2002.

[12] S. F. Hasan, J. Stauder, and A. Tremeau. Optimization of sparse color correspondences for color mapping. In Color and Imaging Conference, pages 128-134, 2012.

[13] K. He, J. Sun, and X. Tang. Single image haze removal using dark channel prior. IEEE Transactions on Pattern Analysis and Machine Intelligence, 33(12):2341-2353.

[14] S. Kagarlitsky, Y. Moses, and Y. Hel-Or. Piecewiseconsistent color mappings of images acquired under various conditions. In IEEE International Conference on Computer Vision, pages 2311-2318, 2009.

[15] H. Koschmieder. Theorie der horizontalen Sichtweite: Kontrast und Sichtweite. Keim \& Nemnich, 1925.

[16] J. Li, K. A. Skinner, R. M. Eustice, and M. JohnsonRoberson. Watergan: Unsupervised generative network to enable real-time color correction of monocular underwater images. IEEE Robotics and Automation Letters, 3(1):387394, 2018.

[17] H. Lu, Y. Li, and S. Serikawa. Underwater image enhancement using guided trigonometric bilateral filter and fast automatic color correction. In 2013 IEEE International Conference on Image Processing, pages 3412-3416, 2013.

[18] R. M. H. Nguyen, S. J. Kim, and M. S. Brown. Illuminant aware gamut-based color transfer. Computer Graphics Forum, 33(7):319-328, Oct. 2014.

[19] M. Panahpour Tehrani, A. Ishikawa, S. Sakazawa, and A. Koike. Iterative colour correction of multicamera systems using corresponding feature points. J. Vis. Comun. Image Represent., 21(5-6):377-391, July 2010.

[20] F. Pitie, A. C. Kokaram, and R. Dahyot. Automated colour grading using colour distribution transfer. Computer Vision and Image Understanding, 107(1-2):123 - 137, 2007. 


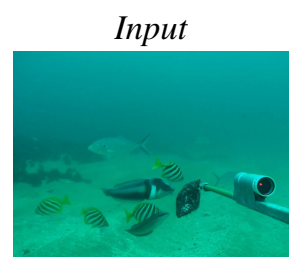

Ours

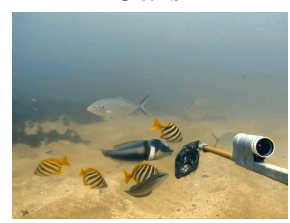

Pitie et al. [20]

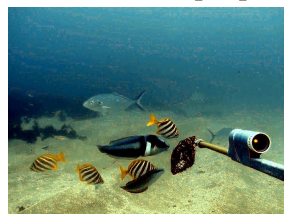

Photoshop

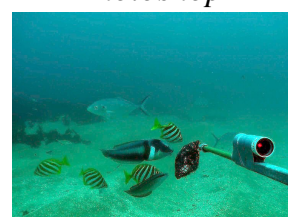

Ancuti et al. [1]

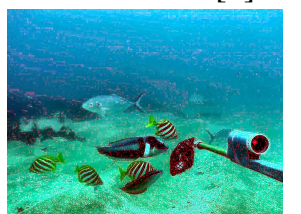

Drews et al. [5]

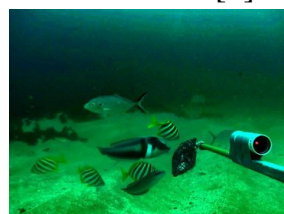

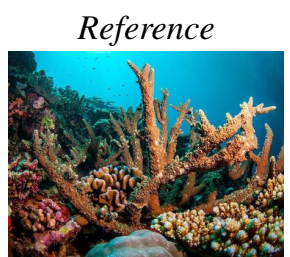

Reinhard et al. [22]

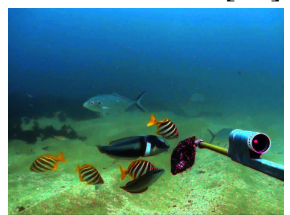

Xiao et al. [25]

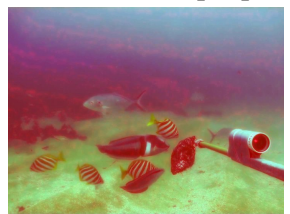

Nguyen et al. [18]

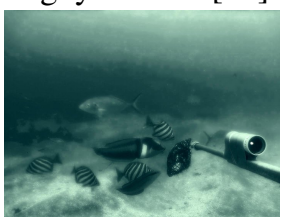

Fu et al. [9]

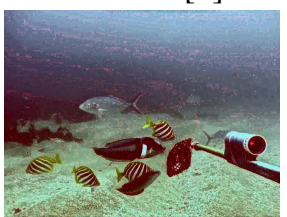

Fabbri et al. [6]

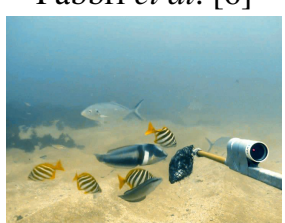

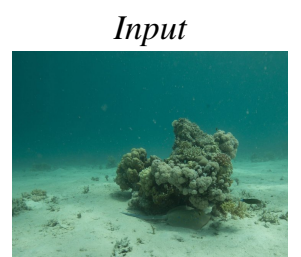

Ours

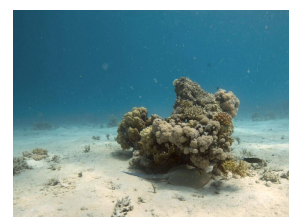

Pitie et al. [20]

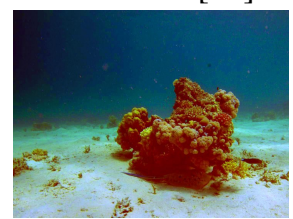

Photoshop

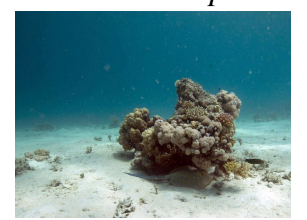

Ancuti et al. [1]

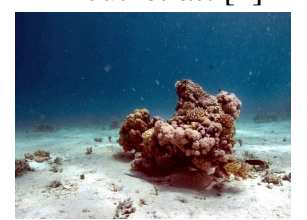

Drews et al. [5]

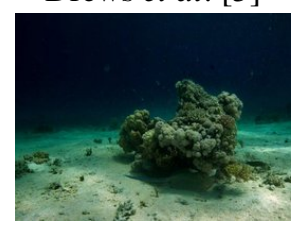

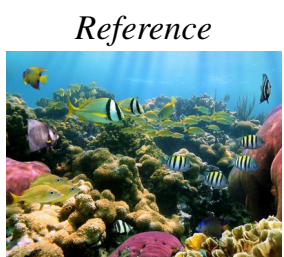

Reinhard et al. [22]

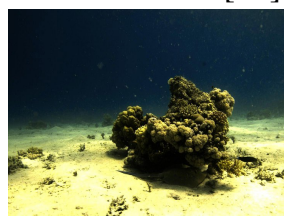

Xiao et al. [25]

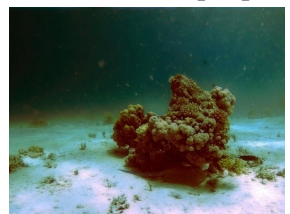

Nguyen et al. [18]

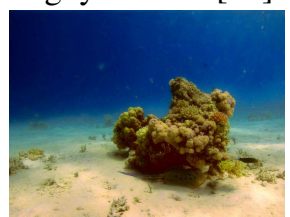

Fu et al. [9]

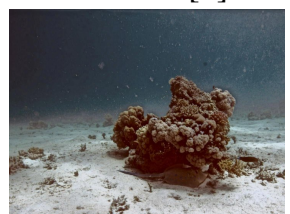

Fabbri et al. [6]

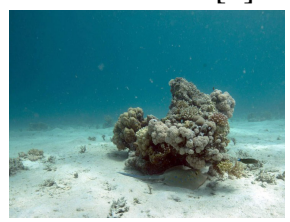

Figure 6. Our approach results compared against traditional color transfer methods and specified underwater color correction methods (below the horizontal line) for underwater image examples.

[21] R. Protasiuk and W. Skarbek. Color transfer by fitting clouds of color points. In Proc.SPIE 10445 Photonics Applications in Astronomy, Communications, Industry, and High Energy Physics Experiment, page 9, 2017.

[22] E. Reinhard, M. Adhikhmin, B. Gooch, and P. Shirley. Color transfer between images. IEEE Computer graphics and applications, 21(5):34-41, 2001.

[23] E. Reinhard and T. Pouli. Colour spaces for colour transfer. In Computational Color Imaging - Third International Workshop, CCIW 2011. Proceedings, pages 1-15, 2011.

[24] X. Xiao and L. Ma. Color transfer in correlated color space. ACM International Conference on Virtual Reality Continuum and Its Applications, 13(2):117-26, 2006.

[25] X. Xiao and L. Ma. Gradient-Preserving Color Transfer. Computer Graphics Forum, 2009.

[26] M. Yang and A. Sowmya. An underwater color image quality evaluation metric. IEEE Transactions on Image Processing, 24(12):6062-6071, 2015. 